\title{
X-ray absorption spectroscopy and atomic force microscopy study of bias-enhanced nucleation of diamond films
}

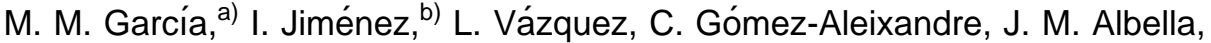 \\ and O. Sánchez \\ Instituto de Ciencia de Materiales, C.S.I.C., Cantoblanco 28049, Madrid, Spain \\ L. J. Terminello \\ Lawrence Livermore National Laboratory, Livermore, California 94551 \\ F. J. Himpsel \\ Department of Physics, University of Wisconsin-Madison, Madison, Wisconsin 53706
}

(Received 22 October 1997; accepted for publication 27 February 1998)

\begin{abstract}
The bias-enhanced nucleation of diamond on $\mathrm{Si}(100)$ has been studied by x-ray absorption near-edge spectroscopy (XANES) and atomic force microscopy, two techniques well suited to characterize nanometric crystallites. Diamond nuclei of $\sim 15 \mathrm{~nm}$ are formed after 5 min of bias-enhanced treatment. The number of nuclei and its size increases with the time of application of the bias voltage. A nanocrystalline diamond film is attained after $20 \mathrm{~min}$ of bias-enhanced nucleation. At the initial nucleation stages, the $\mathrm{Si}$ substrate appears covered with diamond crystallites and graphite, without $\mathrm{SiC}$ being detected by XANES. (C) 1998 American Institute of Physics. [S0003-6951(98)02117-2]
\end{abstract}

Diamond nucleation is a key step in the growth of diamond thin films on Si wafers, because it affects strongly the growth rate, film morphology, and film quality. The application of a dc bias as a pretreatment is a common procedure to promote the formation of diamond nuclei on a Si surface. ${ }^{1}$ However, the mechanism of this bias-enhanced nucleation $(\mathrm{BEN})$ is still controversial. ${ }^{2-6}$

The nanometric size of the diamond nuclei makes common characterization techniques in thin-film growth ambiguous. Scanning electron microscopy (SEM) resolution is often larger than the nuclei size. Raman spectroscopy presents problems due to wave-vector uncertainty. ${ }^{7,8}$ Transmission electron microscopy (TEM) lacks surface sensitivity. To overcome these problems we have chosen atomic force microscopy (AFM) and $\mathrm{x}$-ray absorption near-edge structure (XANES).

Among the advantages of AFM are its high dynamic range, high resolution, the capability to evaluate the surface roughness, and the possibility to study nonconductive samples. With regards to XANES, the technique is very sensitive to the local order in the material, is not limited by the size of the crystallites, it does not require good conductive samples, yields very different spectra for graphite and diamond phases, and the absorption cross sections for carbon atoms with $s p^{2}$ and $s p^{3}$ hybridizations are similar.

The diamond films were grown in a $2.45 \mathrm{GHz}$ ASTEX microwave plasma-assisted chemical vapor deposition (CVD) system. The substrates were 2 in. (100) mirrorpolished $\mathrm{Si}$ wafers, that were immersed in a methane and hydrogen plasma during the whole process. Thin-film growth was performed in two separate stages with different condi-

${ }^{a)}$ Electronic mail: marpoza@icmm.csic.es

${ }^{b)}$ Also at Lawrence Livermore National Laboratory, Livermore, California 94551. tions. In the first stage of BEN pretreatment, nucleation was favored by application of a negative dc bias voltage of $250 \mathrm{~V}$ to the substrate in a mixture of $4 \% \mathrm{CH}_{4}$ in $\mathrm{H}_{2}$ at a pressure of 17 Torr, with a MW power of $450 \mathrm{~W}$, and the substrate held at $850^{\circ} \mathrm{C}$. Four samples A, B, C, and D, corresponding to 5 , 10,15 , and 20 min of pretreatment time, respectively, were prepared. In the second stage, growth was favored for $1 \mathrm{~h}$ at 40 Torr pressure, $1200 \mathrm{~W}$ power, and $0.5 \%$ methane in hydrogen. Identical growth conditions were used for a set of samples labeled $\mathrm{A}^{\prime}, \mathrm{B}^{\prime}, \mathrm{C}^{\prime}$, and $\mathrm{D}^{\prime}$ corresponding to BEN pretreatment times of 5, 10, 15, and 20 min, respectively.

The morphology of the films was characterized by SEM, using a HITACHI SC-410 microscope, and by AFM using a Nanoscope III system operating in the tapping mode at ambient conditions with silicon cantilevers. The SEM images (not shown) obtained from samples $\mathrm{A}^{\prime}-\mathrm{D}^{\prime}$ reveal faceted crystals indicating a certain influence of the nucleation time on the film surface morphology, although it is difficult to obtain quantitative data. However, it is possible to obtain the average grain size and the root-mean-square (rms) roughness values directly from the AFM images by the standard AFM software.

The Raman spectra were obtained with a DILOR $x-y$ Raman spectrometer in the range $1200-1800 \mathrm{~cm}^{-1}$. The quantitative analysis of the spectra has been performed by fitting the experimental spectra to a sum of Lorentzian and Gaussian curves as described previously. ${ }^{9}$

The XANES experiments were performed at the Beamline 8.2 of the Stanford Synchrotron Radiation Laboratory (SSRL). The data were collected in the total yield mode by recording the sample current to ground. Simultaneously, the signal from a gold grid located upstream in the x-ray path was recorded for normalization purposes. A second normalization was performed by recording the signal from a clean $\mathrm{Si}(100)$ wafer in the energy region under study, to eliminate 


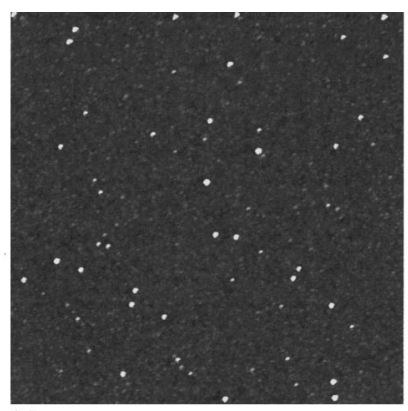

(a)

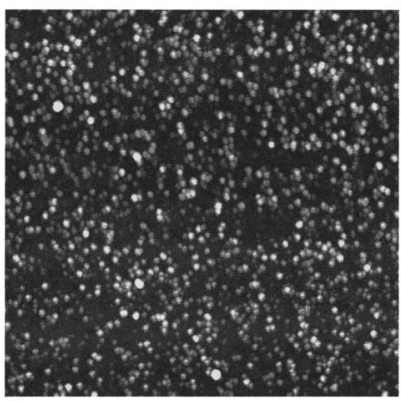

(c)

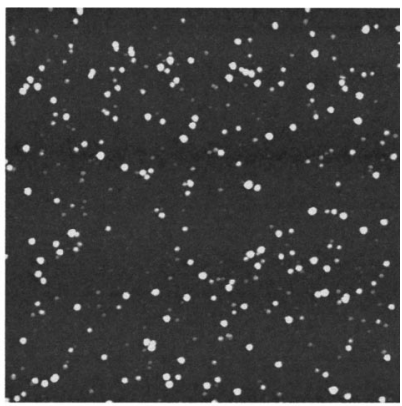

(b)

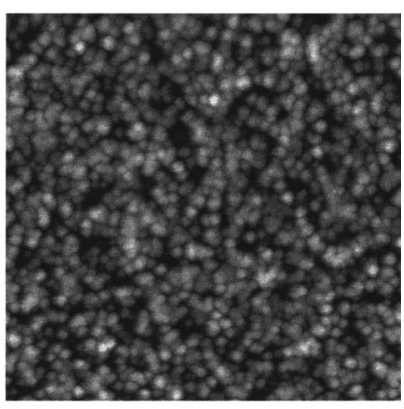

(d)

FIG. 1. $4 \times 4 \mu \mathrm{m}^{2}$ tapping-mode AFM images of bias-enhanced pretreated samples. Images (a)-(d) represent samples A-D, which correspond to biasenhanced nucleation times of 5, 10, 15, and $20 \mathrm{~min}$, respectively. In pictures (a) -(c) the diamond nuclei appear as bright dots. Picture (d) shows a granular continuous diamond film

the absorption structure due to contamination in the beamline optics. Reference spectra were measured from a standard type IIa diamond, a highly oriented pyrolitic graphite (HOPG), and a $\beta$-SiC single-crystal sample. All samples were outgassed in vacuum to about $400{ }^{\circ} \mathrm{C}$ previously to the XANES measurements to eliminate the surface contamination.

Figures 1(a)-1(d) show AFM images of samples A-D, respectively, for which only the pretreatment stage was performed. Nuclei are clearly observed as protrusions on the flat silicon surface. After $5 \mathrm{~min}$ of bias pretreatment nuclei, around $50 \mathrm{~nm}$ wide and $15 \mathrm{~nm}$ high, are already formed. The nuclei diameter could be overestimated because of the tip radius-typically, $10-30 \mathrm{~nm}$ - being tip convolution effects relatively more important when the nuclei imaged are smaller. In contrast, the nuclei height is a reliable measurement since it is not distorted by the tip geometry. Figures 1(a)-1(c) evidence that the nuclei density increases with the bias pretreatment time. It is also observed that after $20 \mathrm{~min}$ [Fig. 1(d)] of bias treatment, most of the nuclei coalesce forming a continuous film (see Table I).

The AFM images from samples $\mathrm{A}^{\prime}-\mathrm{D}^{\prime}$, for which

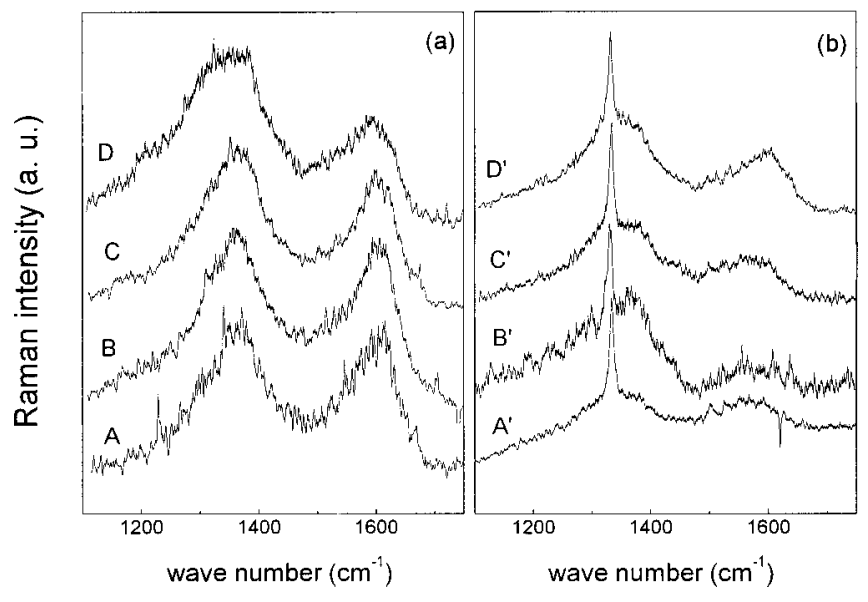

FIG. 2. (a) Raman spectra of BEN pretreated samples A-D. (b) Raman spectra of samples $\mathrm{A}^{\prime}-\mathrm{D}^{\prime}$ with similar pretreatment to $\mathrm{A}-\mathrm{D}$ plus $1 \mathrm{~h}$ diamond growth.

growth was performed on pretreated substrates, show granular faceted films. The data derived from the images are presented in Table I, indicating that the film surface roughness and average grain size decrease as the biasing time increases. This fact implies that the grain size is inversely correlated with the nucleation density. These data show that in order to obtain flat diamond films it is convenient to employ large nucleation times.

Raman spectra from all the samples are presented in Fig. 2. The diamond characteristic peak at $1332 \mathrm{~cm}^{-1}$ is only observed for samples $\mathrm{A}^{\prime}-\mathrm{D}^{\prime}$. For the pretreated samples $\mathrm{A}-\mathrm{D}$, only peaks corresponding to microcrystalline graphite (1340 and $\left.1390 \mathrm{~cm}^{-1}\right)$ and amorphous carbon $\left(1500 \mathrm{~cm}^{-1}\right)$ appear. ${ }^{9}$ According to the Raman spectra, samples $\mathrm{A}^{\prime}-\mathrm{D}^{\prime}$ would contain about $80 \%$ diamond and $20 \%$ graphite carbon, and samples A-D would contain no diamond. However, Raman spectroscopy is not sensitive to nanometric grains, ${ }^{8}$ and the information it yields comes mainly from the bulk of the sample. The complementary XANES study described below shows that the graphitic phases in samples $\mathrm{A}^{\prime}-\mathrm{D}^{\prime}$ are buried beneath the diamond film.

Figure 3 displays the XANES spectra corresponding to samples $\mathrm{A}-\mathrm{D}$ and $\mathrm{A}^{\prime}-\mathrm{D}^{\prime}$. Reference spectra from natural diamond, HOPG, and $\beta$-SiC samples are also shown. The HOPG spectrum presents two characteristic peaks at 285 and $292 \mathrm{eV}$, due to resonances in the $1 s \rightarrow \pi^{*}$ and $1 s \rightarrow \sigma^{*}$ transitions, respectively. The diamond spectrum presents the absorption edge at $289 \mathrm{eV}$ and a dip at $303 \mathrm{eV}$, which corresponds to a second absolute gap in the diamond band structure. ${ }^{10}$ The $\beta$-SiC spectrum is complex and composed of

TABLE I. Data obtained from the AFM images.

\begin{tabular}{|c|c|c|c|c|c|c|c|}
\hline \multicolumn{5}{|c|}{ Pretreatment } & \multicolumn{3}{|c|}{ Pretreatment $+1 \mathrm{~h}$ growth } \\
\hline Sample & $\begin{array}{l}\text { Bias time } \\
\quad(\min )\end{array}$ & $\mathrm{Nuclei} / \mu \mathrm{m}^{2}$ & $\begin{array}{l}\text { Average nucleus } \\
\text { size }(\mathrm{nm})\end{array}$ & $\begin{array}{c}\text { Average nucleus } \\
\text { height }(\mathrm{nm})\end{array}$ & Sample & $\begin{array}{l}\text { Roughness rms } \\
\qquad(\mathrm{nm})\end{array}$ & $\begin{array}{l}\text { Average grain } \\
\text { size }(\mathrm{nm})\end{array}$ \\
\hline A & 5 & 5 & $46 \pm 13$ & $12 \pm 4$ & $\mathrm{~A}^{\prime}$ & 70 & 450 \\
\hline B & 10 & 25 & $49 \pm 20$ & $36 \pm 8$ & $\mathrm{~B}^{\prime}$ & 56 & 375 \\
\hline $\mathrm{C}^{\mathrm{a}}$ & 15 & 70 & $65 \pm 33$ & $20 \pm 5$ & $\mathrm{C}^{\prime}$ & 54 & 300 \\
\hline $\mathrm{D}$ & 20 & Film & Film & Film & $\mathrm{D}^{\prime}$ & 15 & 120 \\
\hline
\end{tabular}

${ }^{a}$ In this case, because of the collapse of the nuclei the final average nucleus size is overestimated whereas that of the nuclei density is underestimated. 


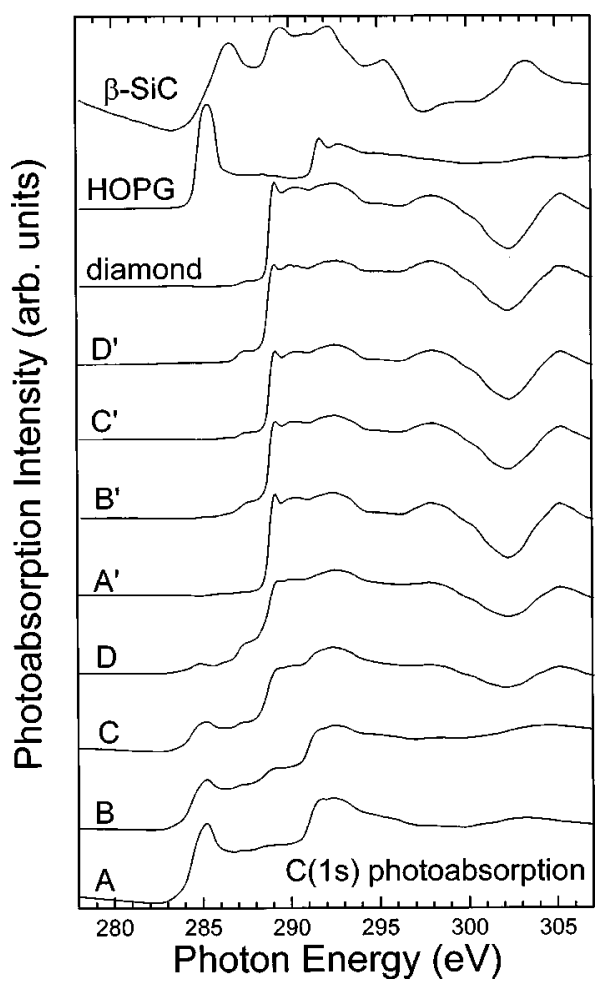

FIG. 3. C (1s) XANES spectra of BEN pretreated samples A-D with diamond nuclei, samples $\mathrm{A}^{\prime}-\mathrm{D}^{\prime}$ with diamond film growth, and reference spectra from diamond, graphite (HOPG) and $\beta$-SiC.

several peaks in the $283-303 \mathrm{eV}$ region. The XANES spectrum from sample A exhibits the typical features of graphite, although wider than the HOPG reference due to the amorphous character of this graphitic phase. A small feature at $289 \mathrm{eV}$ is present, although hardly noticeable in the scale of the figure, that coincides with the diamond absorption edge. The spectrum obtained from sample B is similar, but now the diamond feature is clearly observed. For sample $\mathrm{C}$, the diamond edge dominates and the dip at $303 \mathrm{eV}$ is almost developed. Finally, the spectrum representing the D sample is similar to the diamond reference, except for the absence of a well-resolved excitonic peak at the absorption edge. No features resembling $\beta$-SiC are observed. The $s p^{3}$-bonded carbon content for each sample was estimated from the relative height of the $\sigma^{*}$ absorption edge in diamond-at $290 \mathrm{eV}-$ and graphite-at $292 \mathrm{eV}$. Accordingly, the diamond content at the surface is $5 \%, 30 \%, 75 \%$, and $90 \%$ for $\mathrm{A}, \mathrm{B}, \mathrm{C}$, and D samples, respectively.

Samples $\mathrm{A}^{\prime}-\mathrm{D}^{\prime}$ present similar XANES spectra, with the characteristic features of diamond and without any graphitic feature. The peak at $288 \mathrm{eV}$ observed in samples B' and $\mathrm{D}^{\prime}$ is due to $\mathrm{C}-\mathrm{H}$ bonding and disappears with vacuum annealing. The absence in the other two samples is due to slightly different outgassing times and temperatures.

The AFM and XANES results show that moderate nucleation treatments produce diamond nuclei. The number of nuclei increases with the pretreatment time until a continuous diamond film is attained. The continuous diamond film formed by BEN is hardly detected by Raman spectroscopy due to the nanometric crystallite size. This is of practical interest from the tribological standpoint because small grain diamond films have low friction coefficients. ${ }^{11}$

The XANES spectra of the pretreated samples do not Downloaded 24 Feb 2010 to 161.111.180.191. Redistribution subject show any feature related to $\mathrm{SiC}$, which some studies have related to the diamond nucleation. Because of the surface sensitivity of XANES, it would be possible that a SiC layer is buried beneath the graphite phase. However, in that case, this $\mathrm{SiC}$ layer would not be directly related to the diamond nucleation.

Our data show that graphite and diamond phases coexist from short nucleation times (i.e., $5 \mathrm{~min}$ ), the graphitic phase content being reduced as the nucleation time increases. This scenario is compatible with the formation of diamond nuclei on top of the graphitic layer in agreement with Robertson's subplantation model. ${ }^{12}$ However, from our XANES results it is not sure that a continuous graphite layer is formed before the diamond nucleation. It is possible that diamond nuclei are formed directly on silicon simultaneously with the formation of graphite. In fact, the direct growth of diamond on $\mathrm{Si}$ has been reported from TEM observations of samples prepared under similar conditions. ${ }^{13}$

In conclusion, by combining AFM and XANES we prove that the grains observed at the initial stages of biasenhanced nucleation are diamond nuclei. No $\mathrm{SiC}$ is detected at any stage of the nucleation process. Moderate nucleation pretreatment times (in the 5-20 min range) induce formation of a high diamond nuclei density. A correlation between the initial nucleation density and the final surface roughness of the diamond film grown is found. Long nucleation times result in formation of a continuous film composed of nanometric diamond crystallites.

This work has been partially financed by the Spanish CICYT under Project Nos. MAT96-0529 and PB94-53, and by the U.S. Office of Basic Energy Science, DOE, through the Lawrence Livermore National Laboratory under Contract No. W-7405-ENG-48, and the Advanced Light Source, LBNL, under Contract No. DE-AC03-76SF00098. Two of the authors (M.M.G. and I.J) acknowledge support from the Spanish Ministerio de Educación y Cultura.

${ }^{1}$ S. Yugo, T. Kania, T. Kimura, and T. Muto, Appl. Phys. Lett. 58, 1036 (1991).

${ }^{2}$ B. R. Stoner, G.-H. Ma, S. D. Wolter, and J. T. Glass, Phys. Rev. B 45, 11067 (1992).

${ }^{3}$ X. Jiang, K. Schiffmann, and C.-P. Klages, Phys. Rev. B 50, 8402 (1994).

${ }^{4}$ J. Robertson, J. Gerber, S. Sattel, M. Weiler, K. Jung, and H. Ehrhardt, Appl. Phys. Lett. 66, 3287 (1995).

${ }^{5}$ P. Reinke, P. Kania, P. Oelhafen, and R. Guggenheim, Appl. Phys. Lett. 68, 22 (1996).

${ }^{6}$ T.-Y. Seong, D.-G. Kim, K.-K. Choi, and Y. J. Baik, Appl. Phys. Lett. 70, 3368 (1997).

${ }^{7}$ R. J. Nemanich, S. A. Solin, and R. M. Martin, Phys. Rev. B 23, 6348 (1981).

${ }^{8}$ D. M. Gruen, A. R. Krauss, D. Zuiker, R. Csencsits, L. J. Terminello, J. A. Carlisle, I. Jimenez, D. G. J. Sutherland, D. K. Shuh, W. Tong, and F. J. Himpsel, Appl. Phys. Lett. 68, 1640 (1996).

${ }^{9}$ M. M. García Poza, M. Hernández Vélez, J. Jiménez, C. GómezAleixandre, J. Sánchez Olías, A. Blanco Montes, and J. M. Albella, Mater. Lett. 29, 111 (1996).

${ }^{10}$ J. F. Morar, F. J. Himpsel, G. Hollinger, G. Hughes, and J. L. Jordan, Phys. Rev. Lett. 54, 1960 (1985).

${ }^{11}$ D. M. Gruen, A. R. Krauss, C. D. Zuiker, and R. Csencsits, J. Electrochem. Soc. 144, L224 (1997); A. Erdemir, C. Bindel, G. R. Fenske, C. Zuiker, R. Csencsits, A. R. Krauss, and D. M. Gruen, Diamond Films Technol. 6, 31 (1996).

${ }^{12}$ J. Robertson, Diamond Relat. Mater. 2, 984 (1993); 3, 361 (1994).

${ }^{13}$ L. Chang, C. J. Chen, F. R. Chen, S. F. Hu, and T. S. Lin, Diamond Relat. Mater. 5, 326 (1996). 